J.-S. CHA

KODAI MATH. J.

16 (1993). $220-226$

\title{
MARGOLIS HOMOLOGY AND MORAVA $K$-THEORY FOR COHOMOLOGY OF THE DIHEDRAL GROUP
}

\author{
BY JUN-SIM CHA
}

\begin{abstract}
In this paper, we note that the Margolis homology $H\left(H^{*}(B G ; \boldsymbol{Z} / p), Q_{n}\right)$ relates deeply the Morava $K$-theory $K(n)^{*}(B G)$. In particular we compute $K(n)^{*}(B D)$ for the dihedral group $D$ by using Atiyah-Hirzebruch spectral sequence.
\end{abstract}

\section{$\S 0$. Introduction.}

Let $G$ be a finite group and $H^{*}(B G ; Z / p)$ be the cohomology of $G$ with the coefficient $\boldsymbol{Z} / p$ for a prime number $p$. Since the restriction map to a sylow $p$-group $S$ of $G$ is injective, it is important to know the cohomology of $p$-groups. However it seems a very difficult problem to compute $H^{*}(B S ; \boldsymbol{Z} / p)$ when $S$ is a nonabelian $p$-group. In this paper we consider the case $p=2$. The smallest nonabelian 2-groups $S$ have the order $2^{3}$, which have two types $D$ and $Q$; the dihedral and the quaternion groups. The cohomology $H^{*}(B G ; \boldsymbol{Z} / p), G=D, Q$ are determined by Atiyah, Evens respectively [A], [E].

In this paper we first study the Margolis homology $H\left(H^{*}(B D ; \boldsymbol{Z} / 2), Q_{n}\right)$ for the dihedral group $D$ and next study Morava $K$-theory $K(n)^{*}(B D)$ where $K(n)^{*}(-)$ is the cohomology theory with the coefficient $K(n)^{*}=\boldsymbol{Z} / p\left[v_{n}, v_{n}^{-1}\right]$. Such $K(n)^{*}(B D)$ are given by Tezuka-Yagita [T-Y2] using $B P$-theory. However we use here only Atiyah-Hizebruch spectral sequence for $K(n)^{*}$ theory. In particular we correct some inaccuracy of results in Tezuka-Yagita [T-Y2].

Quite recently I. J. Leary decided the muliplicative structure of $H^{*}(B G ; \boldsymbol{Z} / p)$ for groups of order $p^{3}$ [Ly2] by using the cohomology of group $\tilde{G}$ which is the central product of $G$ and 1-dimensional sphere $S^{1}$. The cohomology ring $H^{*}(B D ; \boldsymbol{Z} / 2)$ is very easy. But its Margolis homology seems not so easy. Hence we first study Margolis homology of $H^{*}(B \tilde{D} ; Z / 2)$ and next consider that of $H^{*}(B D ; Z / 2)$. I thank Nobuaki Yagita who introduced me to these problems.

Received October 9, 1992; revised December 21, 1992. 


\section{$\S 1$. The nonabelian $p$-group of the order 8 .} groups

Let $G$ be a nonabelian group of $|G|=8$. Then $G$ is one of the following

$$
\begin{aligned}
& \left.D=\langle a, b| a^{4}=b^{2}=1,[a, b]=a^{2}\right), \text { dihedral group, } \\
& Q=\langle a, b|\left[a^{4}=b^{4}=1,[a, b]=a^{2}=b^{2}\right\rangle, \text { quaternion group. }
\end{aligned}
$$

For each group $G$, there is a central extension

$$
1 \longrightarrow \boldsymbol{Z} / 2 \longrightarrow G \longrightarrow \boldsymbol{Z} / 2 \oplus \boldsymbol{Z} / 2 \longrightarrow 1
$$

which induces the spectral sequence

$$
E_{2}^{*, *}=H^{*}\left(B(\boldsymbol{Z} / 2 \oplus \boldsymbol{Z} / 2 ; \boldsymbol{Z} / 2), H^{*}(B(\boldsymbol{Z} / 2 ; \boldsymbol{Z} / 2))\right) \Longrightarrow H^{*}(B G ; \boldsymbol{Z} / 2) .
$$

where $E_{2}^{*, *}=S_{2} \otimes \boldsymbol{Z} / 2[z]$ and $S_{2}=\boldsymbol{Z} / 2\left[x_{1}, x_{2}\right]$.

It is known that ([Ls], [Q]) that

$$
d_{2} z= \begin{cases}x_{1} x_{2} & \text { for } G=D \\ x_{1}, x_{2}+x_{1}^{2}+x_{2}^{2} & \text { for } G=Q\end{cases}
$$

Then by the Cartan-Serre transgression theorem

$$
d_{3} z^{2}=x_{1}^{2} x_{2}+x_{1} x_{2}^{2} .
$$

Now we consider the case of the dehedral group.

LEMMA 1.2 . When $G=D, H^{*}(B G ; \boldsymbol{Z} / 2) \cong E_{3} \cong S_{2} /\left(x_{1} x_{2}\right) \otimes \boldsymbol{Z} / 2\left[z^{2}\right]$

Proof. We know that $d_{2} z=x_{1} x_{2}$ and $E_{2}^{*} *=\boldsymbol{Z} / 2\left[x_{1}, x_{2}\right] \otimes \boldsymbol{Z} / 2[z]$. Let $a \in$ $\boldsymbol{Z} / 2\left[x_{1}, x_{2}\right]$. Now $d_{2}(a z)=d_{2} a \cdot z+(-1)^{|a|} a \cdot d_{2} z=(-1)^{|a|} a \cdot x_{1} x_{2}$ and $d_{2}\left(a z^{2}\right)=0$. Therefore $\operatorname{Ker} d_{2}\left(E_{2}^{1, *}\right)=0$ and $\operatorname{Im} d_{2}\left(E_{2}^{1, *}\right)=\operatorname{Ideal}\left(x_{1} x_{2}\right)$. Hence $E_{3}^{*, *}=H\left(E_{2}^{*}, *, d_{2}\right)$ $=\boldsymbol{Z} / 2\left[x_{1}, x_{2}\right] /\left(x_{1} x_{2}\right) \otimes \boldsymbol{Z} / 2\left[z^{2}\right]$. Since $d_{3} z^{2}=x_{1}^{2} x_{2}+x_{1} x_{2}^{2}=0 \bmod \left(x_{1} x_{2}\right)$, we have $E_{3}^{*, *} \cong E_{\infty}^{*, *} \quad$ q. e. d.

§2. $I I^{*}(B D ; \boldsymbol{Z} / 2)$.

In this section we calculate the cohomology of the dehedral group $D$ by the another way. Given a finite group $G$ and a central cyclic subgroup $C$, we fix an embedding of $C$ into $S^{1}$, and define $\tilde{G}=G \times{ }_{\langle c\rangle} S^{1}$. Then we have the exact sequence

$$
1 \longrightarrow S^{1} \longrightarrow \tilde{D} \longrightarrow Z / 2 \oplus Z / 2 \longrightarrow 1
$$

which induces the spectral sequence.

$$
E_{2}^{* \cdot *}=H^{*}\left(B(\boldsymbol{Z} / 2 \oplus \boldsymbol{Z} / 2 ; \boldsymbol{Z} / 2), H^{*}\left(B S^{1} ; \boldsymbol{Z} / 2\right)\right) \Longrightarrow H^{*}(B \tilde{D} ; \boldsymbol{Z} / 2),
$$


where $E_{2}^{*, *}=\boldsymbol{Z} / 2\left[x_{1}, x_{2}\right] \otimes \boldsymbol{Z} / 2[u]$ and $d_{3} u=x_{1}^{2} x_{2}+x_{1} x_{2}^{2}$. The $E_{2}$-term is given by

$$
E_{2}^{*, 2 j} \cong \begin{cases}Z / 2\left[x_{1}, x_{2}\right] /\left(d_{3} u\right) & j=0 \bmod 2 \\ \operatorname{Ker}\left(d_{3} u\right) & j=1 \bmod 2\end{cases}
$$

In this paper, let us write $\operatorname{gr} A=F$ if $F=\bigoplus_{\imath=0}^{s} F_{i} / F_{\imath+1}$ for some filtration $A=F_{0} \supset F_{1} \supset \cdots \supset F_{s}$.

THEOREM 2.1. $H^{*}(B \tilde{D} ; \boldsymbol{Z} / 2) \cong E_{3}^{*, *} \cong \boldsymbol{Z} / 2\left[x_{1}, x_{2}\right] /\left(x_{1}^{2} x_{2}+x_{1} x_{2}^{2}\right) \otimes \boldsymbol{Z} / 2\left[u^{2}\right]$.

Proof. If $d_{3}(a u)=a d_{3} u=a\left(x_{1}^{2} x_{2}+x_{1} x_{2}^{2}\right)=0 \quad$ in $Z / 2\left[x_{1}, x_{2}\right]$, where $a \in$ $Z / 2\left[x_{1}, x_{2}\right]$, then $a=0$. Hence $\operatorname{Ker}\left(d_{3} u\right)=0$. Now $d_{5} u^{2}=d_{5} S q^{2} u=S q^{2}\left(x_{1}^{2} x_{2}+x_{1} x_{2}^{2}\right)$

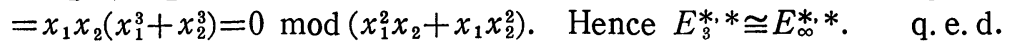

To find $H^{*}(B D ; \boldsymbol{Z} / 2)$, given $H^{*}(B \tilde{D} ; \boldsymbol{Z} / 2)$, we use the Serre spectral of the fibration

$$
S^{1} \longrightarrow B D \longrightarrow B \tilde{D}
$$

This induces the spectral sequence

$$
E_{2}^{*, *}=H^{*}(B \tilde{D} ; Z / 2) \otimes H^{*}\left(S^{1} ; Z / 2\right) \Longrightarrow H^{*}(B D ; Z / 2) .
$$

THEOREM 2.2. Let $z \in H^{1}\left(S^{1} ; Z / 2\right)$ be a generator. Then

$\operatorname{gr} H^{*}(B D ; Z / 2) \cong H^{*}(B \tilde{D} ; Z / 2) /\left(d_{2} z\right) \oplus\left(\operatorname{Ker} d_{2} z\right) \cdot z$

$$
\cong S_{2} \otimes Z / 2\left[u^{2}\right] /\left(x_{1} x_{2}\right) \oplus S_{2} \otimes Z / 2\left[u^{2}\right] /\left(x_{1} x_{2}\right)\left\{\left(x_{1}+x_{2}\right) z\right\}
$$

Proof. First note $d_{2} z=x_{1} x_{2}$. Since $x_{1}^{2} x_{2}+x_{1} x_{2}^{2}=x_{1} x_{2}\left(x_{1}+x_{2}\right), \operatorname{Ker} d_{2}$ is generated by $\left\{\left(x_{1}+x_{2}\right)\right\}$. q. e. $d$.

In section $\S 1$ we know already $H^{*}(B D ; \boldsymbol{Z} / 2) \cong S_{2} \otimes \boldsymbol{Z} / 2[u] /\left(x_{1} x_{2}\right)$. From Theorem 2.2, a filtration of $C=H^{*}(B D)$ is given

$$
\begin{aligned}
& F_{1}=H^{*}(B \tilde{D} ; \boldsymbol{Z} / 2) /\left(x_{1} x_{2}\right) \cong S_{2} \otimes \boldsymbol{Z} / 2\left[u^{2}\right] /\left(x_{1} x_{2}\right) \\
& C / F_{1} \cong \operatorname{Ker} d_{2} z \cong S_{2} \otimes \boldsymbol{Z} / 2\left[u^{2}\right] /\left(x_{1} x_{2}\right)\left\{\left(x_{1}+x_{2}\right) z\right\}
\end{aligned}
$$

with identifying $\left(x_{1}+x_{2}\right) z$ by $u$.

\section{§ 3. Margolis homology of $H^{*}(B D ; \boldsymbol{Z} / 2)$.}

We consider the Margolis homology defined by the Milnor primitive derivation $Q_{n}, H\left(H^{*}(B D ; Z / 2), Q_{n}\right)$. Here $Q_{n}$ is defined by $Q_{n}\left(x_{1}\right)=x_{1}^{2 n+1}, Q_{n}\left(x_{2}\right)=$ $x_{2}^{2 n+1}$. It is known that $u^{2} \in H^{*}(B \tilde{D} ; Z / 2)$ is represent by Chern class. Hence $Q_{n}\left(u^{2}\right)=0$.

Let us denote $u^{2}$ (resp. $\left.x_{1}^{2}, x_{2}^{2}\right)$ by $c$ (resp. $\left.y_{1}, y_{2}\right)$. 
THEOREM 3.1. $H\left(H^{*}(B \tilde{D} ; \boldsymbol{Z} / 2), Q_{n}\right) \cong \boldsymbol{Z} / 2\left[y_{1}, y_{2}, c\right] /\left(y_{1}^{2} y_{2}+y_{1} y_{2}^{2}, y_{1}^{2^{n}}, y_{2}^{2^{2 n}}\right)$

$$
\oplus\left(\boldsymbol{Z} / 2\left[y_{1}=y_{2}, c\right] /\left(y_{1}^{2 n}\right)\right)\left\{x_{1} x_{2}\right\} .
$$

Proof. If $f \in \boldsymbol{Z} / 2\left[x_{1}, x_{2}\right] /\left(x_{1}^{2} x_{2}+x_{1} x_{2}^{2}\right) \otimes \boldsymbol{Z} / 2\left[u^{2}\right]$, then we can write $f=$ $a+b x_{1}+c x_{2}+d x_{1} x_{2}+e x_{1}^{2} x_{2}, \quad$ where $a \in Z / 2\left[x_{1}^{2}, x_{2}^{2}, u^{2}\right] /\left(x_{1}^{4} x_{2}^{2}+x_{1}^{2} x_{2}^{4}\right), \quad b \in$ $\boldsymbol{Z} / 2\left[x_{1}^{2}, u^{2}\right], c \in \boldsymbol{Z} / 2\left[x_{2}^{2}, u^{2}\right], d \in \boldsymbol{Z} / 2\left[x_{1}^{2}=x_{2}^{2}, u^{2}\right], e \in \boldsymbol{Z} / 2\left[x_{1}^{2}=x_{2}^{2}, u^{2}\right]$. Then $Q_{n} f$ $=b x_{1}^{2^{n+1}}+c x_{2}^{2^{n+1}}+d x_{1}^{2^{n+1}} x_{2}+d x_{1} x_{2}^{2^{n+1}}+e x_{1}^{2} x_{2}^{2^{n+1}}$. Here $d x_{1}^{2^{n+1}} x_{2}+d x_{1} x_{2}^{2 n+1}=$ $d\left(x_{1}^{2 n+1} x_{2}+x_{1} x_{2}^{2 n+1}\right)=0 \bmod \left(x_{1}^{2} x_{2}+x_{1} x_{2}^{2}\right)$.

Therefore $\operatorname{Ker} Q_{n}=\left\{a+d x_{1} x_{2}\right\}$ and

$$
\operatorname{Im} Q_{n}=\left\{b x_{1}^{2^{n+1}}+c x_{2}^{2^{n+1}}+e x_{1}^{2}{x_{2}^{2}}^{n+1}=b{x_{1}^{2 n+1}}^{2} c x_{2}^{2^{n+1}}+e\left(x_{1} x_{2}\right) x_{2}^{2^{n+1}}\right\} .
$$

Hence we get $H\left(H^{*}(B \tilde{D} ; \boldsymbol{Z} / 2), Q_{n}\right)=\boldsymbol{Z} / 2\left[y_{1}, y_{2}, c\right] /\left(y_{1}^{2} y_{2}+y_{1} y_{2}^{2}, y_{1}^{2 n}, y_{2}^{2 n}\right)$

$$
\oplus\left(\boldsymbol{Z} / 2\left[y_{1}=y_{2}, c\right] /\left(y_{1}^{2 n}\right)\right)\left\{x_{1}, x_{2}\right\} \text {. q.e. d. }
$$

THEOREM 3.2. $\operatorname{gr} H\left(H^{*}(B D ; \boldsymbol{Z} / 2), Q_{n}\right) \cong\left(\boldsymbol{Z} / 2\left[y_{1}, y_{2}\right] /\left(y_{1}, y_{2}, y_{1}^{2^{n}}, y_{2}^{2^{n}}\right)\right.$ $\left.\otimes \boldsymbol{Z} / 2[c] /\left(y_{1} c^{2^{n-1}}, y_{2} c^{2^{n-1}}\right)\right) \oplus \boldsymbol{Z} / 2[c]\left\{y_{1}^{2^{n}-1} e_{1}=y_{2}^{2^{n}-1} e_{2}\right\}$, where $e_{2}=x_{i}\left(x_{1}+x_{2}\right) z$.

Proof. From Theorem 2.2, we already know $\operatorname{gr} H^{*}(B D ; \boldsymbol{Z} / 2)=$ $H^{*}(B \tilde{D} ; \boldsymbol{Z} / 2) /\left(d_{2} z\right) \oplus\left(\operatorname{Ker} d_{2} z\right) z$. First we compute $H\left(H^{*}(B D ; \boldsymbol{Z} / 2) /\left(x_{1} x_{2}\right), Q_{n}\right)$ and secondary compute $H\left(\left(\operatorname{Ker} x_{1} x_{2}\right) z, Q_{n}\right)$. Using the spectral sequence, we get $H\left(H^{*}(B \tilde{D} ; \boldsymbol{Z} / 2), Q_{n}\right)$ at last.

Let $C=\operatorname{gr} H^{*}(B D ; Z / 2)$ and $F_{1}=H^{*}(B \tilde{D} ; Z / 2) /\left(x_{1} x_{2}\right)$. Then we will prove

$$
\begin{gathered}
H\left(F_{1}, Q_{n}\right) \cong \boldsymbol{Z} / 2\left[y_{1}, y_{2}, c\right] /\left(y_{1} y_{2}, y_{1}^{2^{n}}, y_{2}^{2 n}\right) \\
H\left(C / F_{1}, Q_{n}\right) \cong\left(\boldsymbol{Z} / 2\left[y_{1}, y_{2}, c\right] /\left(y_{1} y_{2}, y_{1}^{2^{n}-1}, y_{2}^{2^{n}-1}\right)\right)\left\{y_{1} z, y_{2} z\right\} .
\end{gathered}
$$

First we will prove (3.3).

If $f \in \boldsymbol{Z} / 2\left[x_{1}, x_{2}, u^{2}\right] /\left(x_{1} x_{2}\right)$, then we can write $f=a+b x_{1}+c x_{2}$ where $a \in$ $\boldsymbol{Z} / 2\left[x_{1}^{2}, x_{2}^{2}, u^{2}\right] /\left(x_{1}^{2} x_{2}^{2}\right), b \in \boldsymbol{Z} / 2\left[x_{1}^{2}, u^{2}\right], c \in \boldsymbol{Z} / 2\left[x_{2}^{2}, u^{2}\right]$. Operate $Q_{n}$ to $f$, then $Q_{n} f=b x_{1}^{2^{n+1}}+c x_{2}^{2^{n+1}}$. Therefore $\operatorname{Ker} Q_{n}=\{a\}$ and $\operatorname{Im} Q_{n}=\left\{b x_{1}^{2^{n+1}}+c x_{2}^{2^{n+1}}\right\}$. Hence we get (3.3).

Next we will prove (3.4).

If $f \in\left(\boldsymbol{Z} / 2\left[x_{1}, x_{2}, u^{2}\right] /\left(x_{1} x_{2}\right)\right)\left\{x_{1}+x_{2}\right\}$, then $f=a\left(x_{1}+x_{2}\right)+b x_{1}\left(x_{1}+x_{2}\right)+c x_{2}\left(x_{1}+x_{2}\right)$ $=a\left(x_{1}+x_{2}\right)+b x_{1}^{2}+c x_{2}^{2}, \quad$ where $a \in \boldsymbol{Z} / 2\left[x_{1}^{2}+x_{2}^{2}, u^{2}\right] /\left(x_{1}^{2} x_{2}^{2}\right), \quad b \in \boldsymbol{Z} / 2\left[x_{1}^{2}, u^{2}\right], \quad c \in$ $\boldsymbol{Z} / 2\left[x_{2}^{2}, u^{2}\right]$. Then $Q_{n} f=a\left(x_{1}^{2^{n+1}}+x_{2}^{2^{n+1}}\right)$. Therefore $\operatorname{Ker} Q_{n}=\left\{\left(b x_{1}+c x_{2}\right)\right.$ $\left.\left\{x_{1}+x_{2}\right\}\right\}, \operatorname{Im} Q_{n}=\left\{a\left(x_{1}^{2^{n+1}}+x_{2}^{2^{n+1}}\right)\right\}$. Hence we get (3.4).

At least we consider the spectral sequence

$$
E_{1}=H\left(F_{1}, Q_{n}\right) \oplus H\left(C / F_{1}, Q_{n}\right) \Longrightarrow H\left(C, Q_{n}\right) .
$$

Now we can prove $Q_{n}\left(y_{i} z\right)=y_{i} u_{1}^{2^{n}},=y_{i} c^{2^{n-1}}$, for $i=1,2$. So we can prove $\operatorname{gr} H\left(C, Q_{n}\right) \cong\left(\boldsymbol{Z} / 2\left[y_{1}, y_{2}\right] /\left(y_{1} y_{2}, y_{1}^{2^{n}}, y_{2}^{2^{n}}\right) \otimes \boldsymbol{Z} / 2[c] /\left(y_{1} c^{2^{n}}, y_{2} c^{2^{n}}\right)\right) \oplus \boldsymbol{Z} / 2[c]\left\{y_{1}^{2^{n}-1} e_{1}\right.$ $\left.=y_{2}^{2^{n}-1} e_{2}\right\}$. q.e.d. 


\section{$\S 4$. Morava $K$-theory.}

The Morava $K$-theory $K(n)^{*}(-)$ is generalized cohomology theory with the coefficient $K(n)^{*}=\boldsymbol{Z} / 2\left[v_{n}, v_{n}^{-1}\right],\left|v_{n}\right|=-2^{n+1}+2$.

We consider the Atiyah-Hirzebruch spectral sequence for Morava $K$-theory

$$
E_{2}^{*, *}=\left(H^{*}\left(X ; K(n)^{*}\right) \Longrightarrow K(n)^{*}(X) .\right.
$$

It is known [Hu], [T-Y] that the differential $d_{2^{n+1-1}}(x)=v_{n} \otimes Q_{n} x$. Hence we get

$$
E_{2}^{*, * 1} \cong K(n)^{*} \otimes H\left(H^{*}(X ; Z / 2), Q_{n}\right)
$$

THEOREM 4.1. $\operatorname{gr} K(n)^{*}(B \widetilde{D}) \cong K(n)^{*} \otimes H\left(H^{*}(B \tilde{D} ; \boldsymbol{Z} / 2), Q_{n}\right)$

Proof. $H\left(H^{*}(B \tilde{D} ; \boldsymbol{Z} / 2), Q_{n}\right)$ is generated by even dimensional elements, hence $E_{2}^{*, *+1} \cong E_{\infty}^{*, *}$. q.e.d.

Ravenel [R] showed that $\operatorname{dim}_{K(n) *} K(n)^{*}(B G)$ is finite for each finite group $G$. Hopkins-Kuhn-Ravenel [H-K-R] defined $K(n)$-theory Euler character $\chi_{n}$ by

$$
\chi_{n}(G)=\operatorname{dim}_{K(n) *} K(n)^{\text {even }}(B G)-\operatorname{dim}_{K(n) *} K(n)^{\text {odd }}(B G) .
$$

For $p$-groups $G$, this Euler character can be described in terms of conjugacy classes of commuting $n$-tuples of elements in $G$,

$\chi_{n}(G)=$ number of $\left\{\left(g_{1}, \cdots, g_{n}\right) \mid\left[g_{\imath}, g_{j}\right]=1, g_{i} \in G\right\} / G$ with the conjugate action $g \cdot\left(g_{1}, \cdots, g_{n}\right) \sim\left(g g_{1} g^{-1}, \cdots, g g_{n} g^{-1}\right)$. They also showed (Lemma 5.3.6 in $[\mathrm{H}-\mathrm{K}-\mathrm{R}])$ that $\chi_{n}$ is computed inductively

$$
\chi_{n}(G)=\sum_{\langle g\rangle} \chi_{n-1}\left(C_{G}(g)\right)
$$

where $\langle g\rangle$ runs over conjugate classes in $G$ and $C_{G}(g)=\{h \in G \mid[h, g]=1\}$ is the centralizer of $g$ in $G$.

Now we consider $K(n)^{*}(B D)$. Recall $H\left(H^{*}(B D ; Z / 2), Q_{n}\right)$ in Theorem 3.3. If $d_{r}\left\{y_{1}^{2 n}-1 e_{1}\right\}=0$ for all $r$, then $E_{4}^{*, *} \cong E_{\infty}^{*, *}$. Hence $\operatorname{dim}_{K(n) *} K(n) *(B D)$ is infinite since $c^{s} \neq 0$.

This contradicts the results of Ravenel, therefore we know

$$
d_{r}\left\{y_{1}^{2 n-1} e_{1}\right\}=v_{n}^{k} c^{s} \text { for some } s \text { with } 2\left(2^{n}-1\right)(k+1)+4=4 s .
$$

From Theorem 3.2, $E_{r+1}^{* * *}$ is generated by even dimensional elements. Hence $E_{r+1}^{*, *} \cong E_{\infty}^{* * *}$.

LEMMA 4.5. $\operatorname{dim}_{K(n) *} K(n) *(B D)=2^{2 n}-2^{n}+s$.

Proof. From Theorem 3.2, $K(n)^{*}(B D)$ has $K(n)^{*}$-basis $\left\{y_{1}^{k}, y_{2}^{k}\right\} \otimes c^{j} \oplus c^{h}$ $\left(1 \leqq k<2^{n}, 0 \leqq j<2^{n-1}, 0 \leqq h<s\right)$. Hence we see $\operatorname{dim}_{K(n) *} K(n)^{*}(B D)=2\left(2^{n}-1\right) \times$ $2^{n-1}+s$. q.e.d. 
LEMMA 4.6. $\chi_{n}(D)=2^{2 n}+2^{2 n-1}-2^{n-1}$.

Proof. The conjugacy classes of $D$ are $\langle 1\rangle,\left\langle a^{2}\right\rangle,\left\langle a^{2} b^{j}\right| 0 \leqq i j \leqq 1(i, j) \neq$ $(0,0)\rangle$ and their centralizer are $D, D, \boldsymbol{Z} / 2 \oplus \boldsymbol{Z} / 2$ respectively. So from (4.3)

$$
\begin{aligned}
\chi_{n}(D) & =\sum_{\langle g\rangle} \chi_{n-1}\left(C_{G}(g)\right) \\
& =\chi_{n-1}\left(C_{G}(1)\right)+\chi_{n-1}\left(C_{G}\left(a^{2}\right)\right)+\chi_{n-1}\left(C_{G}(a)\right)+\chi_{n-1}\left(C_{G}(b)\right)+\chi_{n-1}\left(C_{G}(a b)\right) \\
& =\chi_{n-1}(D)+\chi_{n-1}(D)+\chi_{n-1}(\boldsymbol{Z} / 4)+\chi_{n-1}(\boldsymbol{Z} / 2 \otimes \boldsymbol{Z} / 2)+\chi_{n-1}(\boldsymbol{Z} / 2 \otimes \boldsymbol{Z} / 2) \\
& =2 \chi_{n-1}(D)+3 \cdot 2^{2 n-2} .
\end{aligned}
$$

We put $\chi_{n-1}(D)=2^{2 n-2}+2^{2 n-3}-2^{n-2}$. Then $2 \chi_{n-1}(D)+3 \cdot 2^{2 n-2}=2\left(2^{2 n-2}+2^{2 n-3}-2^{n-2}\right)$ $+3 \cdot 2^{2 n-2}=2^{2 n}+2^{2 n-1}-2^{n-1}$. Hence we get this Lemma. q.e.d.

From Lemma 4.5 and Lemma 4.6, we know $s=2^{2 n-1}+2^{n-1}$, hence $k=2^{n}+1$.

THEOREM 4.7. $\operatorname{gr} K(n)^{*}(B D) \cong K(n)^{*}\left(S_{2}^{\prime} /\left(y_{1} y_{2}, y_{1}^{2^{n}}, y_{2}^{2 n}\right)\right)$

$$
\otimes \boldsymbol{Z} / 2[c] /\left(y_{1} c^{2^{n-1}}, y_{2} c^{2^{n-1}}, c^{2^{2 n-1}+2^{n-1}}\right) \text { with } c=u^{2} \text {. }
$$

Remark. The multiplicative structure of $K(n) *(B D)$ was given in Theorem 4.2 in [T-Y]. There were some errors, which were corrected in [T-Y3]. The ring structure is

$$
\begin{aligned}
& K(n)^{*}(B D) \\
& \cong K(n)^{*}\left(S^{\prime} \otimes \boldsymbol{Z} / 2[c] /\left(y_{1}^{2^{n}}, y_{2}^{2^{n}}, v_{n}^{2} c^{2^{n}}=v_{n} c^{2^{n-1}} y_{1}=v_{n} c^{2^{n-1}} y_{2}=y_{1} y_{2}\right) .\right.
\end{aligned}
$$

This consists with ours as following and from (4.8) we deduce

$$
\begin{aligned}
0 & =y_{1}^{2 n} y_{2}=v_{n} y_{1}^{2^{n}-1} c^{2^{n-1}} y_{2}=\cdots=v_{n}^{2 n}\left(c^{2^{n-1}}\right)^{2^{n}} y_{2}=v_{n}^{2^{n}} c^{2^{n-1}\left(2^{n}-1\right)} c^{2^{n-1}} y_{2} \\
& =v_{n}^{2^{n}-1} c^{2^{n-1}\left(2^{n}-1\right)} y_{1} y_{2}=v_{n}^{2^{n}+1} c^{\left(2^{n}-1\right) 2}{ }^{n-1} c^{2^{n}}=v_{n}^{2^{n}+1} c^{2^{2 n-1+2}{ }^{n-1}} .
\end{aligned}
$$

\section{REFERENCES}

[A] M.F. A тigah, Character and cohomology of finite groups, Publ. I.H.E.S., 9 (1961), 23-64.

[A-M] J.E. AdAms And H. R. MARgolis, Module over the Steenrod algebra, Topplogy 10 (1971), 271-282.

[E] L. Evens, On the Chern class of representation of finite groups, Trans. Amer. Math. Soc., 115 (1965), 180-193.

[H-K-R] M. Hopkins, N. KuHn AND D. Ravenel, Generalized group characters and complex oriented cohomology theory, Preprint.

[Hu] J. Hunton, The Morava $K$-theories of wreath product, Math. Proc. Cambridge Phile. Soc., 107 (1990), 309-318. 
[L] G. LEwIS, The integral cohomology rings of groups of order $p^{3}$, Trans. Amer. Math. Soc., 132 (1968), 501-529.

[Ly1] I. J. Leary, The integral cohomolgy rings of some p-groups, Math. Proc. Cambridge Phil. Soc., 110 (1991), 25-32.

[Ly2] I. J. LEARY, The cohomology of certain finite groups, Thesis at the Univ. Cambridge 1990.

[R] D.C. RAVENEL, Morava $K$-theories and finite groups, Symposium on Algebraic Topology in Honor of José Adem, Amer. Math. Soc. (1982), 289-292.

T.Y 1] M. Tezuka And N. Yagita, The varieties of the mod $p$ cohomology rings of extra special p-groups for an odd prime $p$, Math. Proc. Cambridge Phil. Soc., 94 (1983), 449-459.

[T-Y 2] M. Tezuka And N. Yagita, Cohomology of finite groups and the BrownPeterson cohomology, Springer LNM, 1370 (1989), 396-408.

[T-Y 3] M. TEzUKA AND N. Yagita, Complex $K$-Theory of $\operatorname{BSL}_{3}(\boldsymbol{Z})$, to appear in $K$-Theory.

Department of Mathematics

KYUNG-HEE UNIVERSITY

SUWON 449-701

SEOUL, KOREA 